\title{
SEPIA - a new single pixel receiver at the APEX telescope
}

\author{
V. Belitsky ${ }^{1,2}$, I. Lapkin ${ }^{1,2}$, M. Fredrixon ${ }^{1,2}$, D. Meledin ${ }^{1,2}$, E. Sundin ${ }^{1,2}$, B. Billade ${ }^{2}$, S.-E. Ferm ${ }^{1,2}$,

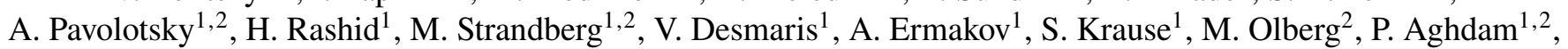 \\ S. Shafiee ${ }^{1,2}$, P. Bergman' ${ }^{2}$ E. De Beck ${ }^{2}$, H. Olofsson ${ }^{2}$, J. Conway², C. De Breuck ${ }^{3}$, K. Immer ${ }^{3}$, P. Yagoubov ${ }^{3}$, \\ F. M. Montenegro-Montes ${ }^{4}$, K. Torstensson ${ }^{4}$, J.-P. Pérez-Beaupuits ${ }^{4}$, T. Klein ${ }^{4}$, W. Boland ${ }^{5}$, A. M. Baryshev ${ }^{5}$, \\ R. Hesper ${ }^{5}$, J. Barkhof ${ }^{5}$, J. Adema ${ }^{5}$, M. E. Bekema ${ }^{5}$, and A. Koops ${ }^{5}$ \\ ${ }^{1}$ Group for Advanced Receiver Development (GARD), Department of Space, Earth and Environment, \\ Chalmers University of Technology, 41296 Gothenburg, Sweden \\ e-mail: victor.belitsky@chalmers.se \\ ${ }^{2}$ Department of Space, Earth and Environment, Chalmers University of Technology, Onsala Space Observatory (OSO), \\ 43992 Onsala, Sweden \\ ${ }^{3}$ European Southern Observatory (ESO), Karl-Schwarzschild-Str. 2, 85748 Garching bei München, Germany \\ ${ }^{4}$ European Southern Observatory, Alonso de Córdova 3107, Vitacura, Casilla 19001, Santiago de Chile, Chile \\ ${ }^{5}$ Netherlands Research School for Astronomy (NOVA), Kapteyn Astronomical Institute, Landleven 12, 9747 AD Groningen, \\ The Netherlands
}

Received 28 June 2017 / Accepted 19 November 2017

\section{ABSTRACT}

\begin{abstract}
Context. We describe the new Swedish-ESO PI Instrument for APEX (SEPIA) receiver, which was designed and built by the Group for Advanced Receiver Development (GARD), at Onsala Space Observatory (OSO) in collaboration with ESO. It was installed and commissioned at the APEX telescope during 2015 with an ALMA Band 5 receiver channel and updated with a new frequency channel (ALMA Band 9) in February 2016.

Aims. This manuscript aims to provide, for observers who use the SEPIA receiver, a reference in terms of the hardware description, optics and performance as well as the commissioning results.

Methods. Out of three available receiver cartridge positions in SEPIA, the two current frequency channels, corresponding to ALMA Band 5, the RF band 158-211 GHz, and Band 9, the RF band 600-722 GHz, provide state-of-the-art dual polarization receivers. The Band 5 frequency channel uses 2SB SIS mixers with an average SSB noise temperature around $45 \mathrm{~K}$ with IF (intermediate frequency) band $4-8 \mathrm{GHz}$ for each sideband providing total $4 \times 4 \mathrm{GHz}$ IF band. The Band 9 frequency channel uses DSB SIS mixers with a noise temperature of $75-125 \mathrm{~K}$ with IF band $4-12 \mathrm{GHz}$ for each polarization.
\end{abstract}

Results. Both current SEPIA receiver channels are available to all APEX observers.

Key words. instrumentation: detectors - techniques: spectroscopic

\section{Introduction}

The Atacama Pathfinder Experiment telescope (APEX), is a collaboration between the Onsala Space Observatory (OSO), the European Southern Observatory (ESO), and the Max-PlanckInstitut für Radioastronomie (MPIfR) to construct and operate a modified ALMA prototype antenna as a single dish on the high altitude site $(5105 \mathrm{~m})$ of the Llano Chajnantor, Chile. The telescope was manufactured by VERTEX Antennentechnik in Duisburg, Germany and is a $12 \mathrm{~m}$ diameter Cassegrain antenna with a surface rms (random mean square) better than $15 \mu \mathrm{m}$, two Nasmyth and a central Cassegrain cabins for instrumentation. Details on the telescope, site, and instruments can be found at http://www.apex-telescope.org/ and in Güsten et al. (2006). The new Swedish-ESO PI Instrument for the APEX telescope (SEPIA) receiver was brought to APEX in the beginning of 2015 via a joint effort from the Group for Advanced Receiver Development (GARD) at OSO, and ESO. GARD performed the optics and cryostat design and construction and the refurbishing of the Band 5 pre-production cartridge (owned by ESO, earlier built by GARD under the European Community FP6 funded project) with the full-production LO system and Warm Cartridge
Assembly, both provided by NRAO via ESO. GARD has built the control system and software, installed the SEPIA receiver in the APEX Cabin A and performed technical commissioning. Initially, the SEPIA receiver contained only an updated ALMA Band 5 pre-production receiver cartridge (Billade et al. 2012) to address the growing interest in future observations with Band 5 at ALMA (first available during cycle 5). The ALMA Band 5 center frequency nearly coincides with the $183.3 \mathrm{GHz}$ water absorption line. The key applications of the Band 5 receivers for ALMA range from observations of the $3_{13}-2_{20} \mathrm{H}_{2} \mathrm{O}$ line at $183.310 \mathrm{GHz}$ in both Galactic objects and nearby galaxies (e.g., Humphreys et al. 2017) to the $158 \mu \mathrm{m}$ emission line of $\mathrm{C}^{+}$from high redshift galaxies (Laing et al. 2010). In the beginning of 2016, SEPIA was equipped with an additional ALMA Band 9 cartridge (Baryshev et al. 2015), providing observers with two state-of-the-art, single-pixel, dual polarization receivers delivering each a total $16 \mathrm{GHz}$ IF band. The realization of the SEPIA receiver project took about one year from the moment the decision was taken, February-March 2014, to the time SEPIA was installed at APEX in February 2015. The success of the SEPIA project was guaranteed by ESO, providing optical windows and filters, access to hardware from ALMA, support from NRAO by 
providing local oscillator (LO), Band 5 warm cartridge assembly (WCA) and FE bias electronics - all purchased by ESO. GARD OSO expertise in designing and constructing mm-wave receivers, in particular the ALMA Band 5 receiver and a long time heritage of APEX instrumentation, allowed the successful completion of the entire project over this short time. In this manuscript, the SEPIA receiver design, the receiver channel performance and the commissioning results are described.

\section{SEPIA receiver design}

\subsection{Optics}

One of the major challenges of bringing the SEPIA receiver with installed ALMA cartridges to the APEX telescope is the necessity to implement tertiary optics, which should not only provide the required and frequency independent illumination of the secondary but also be compatible with the existing optical layout of the APEX Cabin A where all single-pixel heterotion offset from the FE center, the beam tilt offset compensating is different for, for example, ALMA Band $5-2.38^{\circ}$ and for ALMA Band 9 cartridges $-0.93^{\circ}$. SEPIA tertiary optics shall accommodate all these constrains and differences with minimum number of reflecting surfaces (thus minimizing the reflecting loss) and fit a very confined volume within the APEX Cabin A. Specific for SEPIA and in contrast to ALMA FE, we use a rotating cartridge-selection mirror. Such an optical switch addresses limitations of the Nasmyth layout when one receiver channel has access to the sky at a time (Fig. 1). The cartridge-selection mirror (NMF3; Fig. 1) with its precision computer-controlled rotating mechanism facilitates the accommodation of different ALMA cartridges having sdyne receivers are installed. Another serious constraint is a clearance of the APEX Cabin A Nasmyth tube, whose rim is limited by the elevation encoder down to $150 \mathrm{~mm}$ in diameter requiring precision alignment possibilities to avoid Band 5 receiver beam truncation. ALMA receiver cartridges have built-in cold optics optimized for their respective position inside the ALMA Front-End (FE) receiver cryostat place at the antenna focal plane. In particular, depending on the cartridge posipecific differences in the incoming beam positioning as outlined above.

The SEPIA optics have been designed and manufactured with its supporting parts by GARD. The optics is mounted on a separate supporting frame and employs precision mechanical reference devices; these devices were used for initial alignment of the optics with help of a reference laser. Once the optics as a whole is aligned with the tertiary optics of the APEX Cabin A, the reference devices have been locked to allow easier re-installation of the receiver optics and the cryostat without further alignment. The SEPIA tertiary optics unit is normally attached to the APEX Cabin A receiver supporting frame whereas the SEPIA cryostat is attached to the optics-supporting frame. The cryostat-optics frame mechanical interface allows accurate remating of these two parts of the SEPIA receiver without disturbing the optical alignment.

\subsection{SEPIA cryostat}

In order to accommodate the ALMA receiver cartridges at APEX, a compatible cryostat should be used which provides the required mechanical, cryogenic, vacuum, optical and electrical interfaces to the receiver cartridges. The space available in APEX Cabin A and the cabin door opening completely excluded the use of the ALMA FE cryostat. The Nasmyth layout of APEX

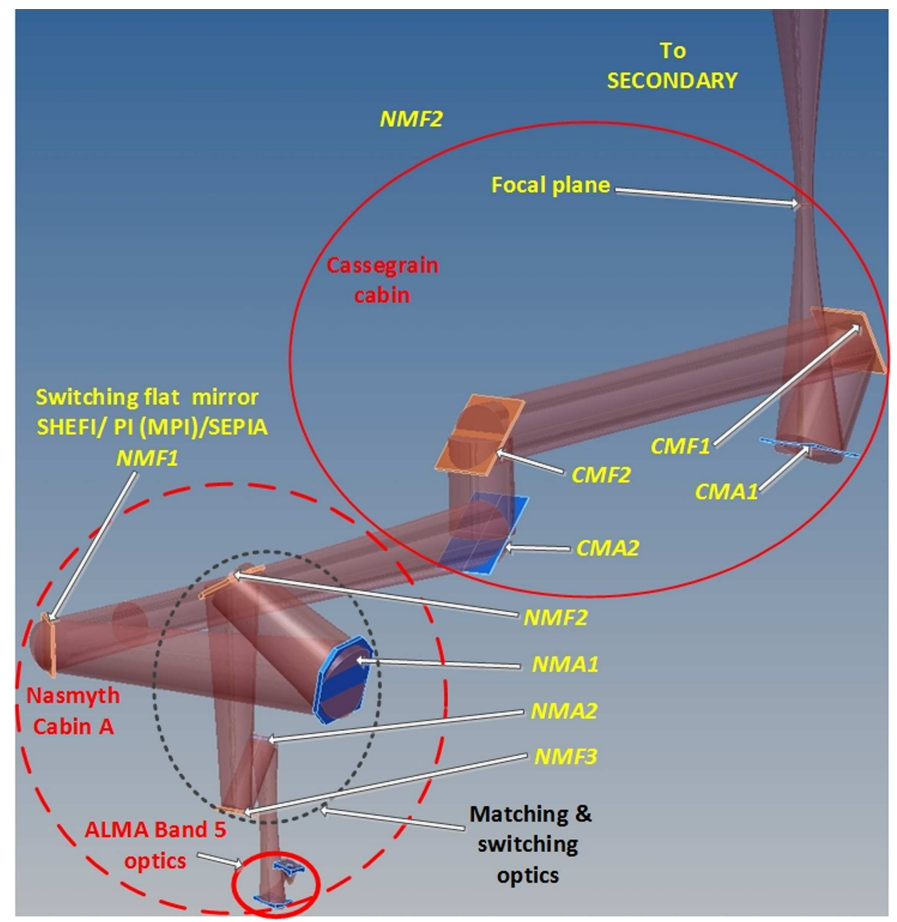

Fig. 1. SEPIA optics (in scale). Mirrors CMF1, CMA1, CMF2, CMA2 and Cabin A Instrument switch, NMF1, are part of earlier existing tertiary optics for the APEX multi-channel facility instrument SHeFI and PI instruments of the APEX Cabin A. Mirrors NMA1, NMF2, NMF3 (SEPIA channel switch), NMA2 are parts of the SEPIA dedicated relay optics. Letters $\mathrm{A}$ and $\mathrm{F}$ in the mirror name indicate active and flat mirrors, respectively. Gaussian beam visualization is done for the lowest ALMA Band 5 RF frequency $158 \mathrm{GHz}$. The entire relay optics (APEX Cabin A+C+SEPIA) provides frequency independent illumination of the secondary with $-12.5 \mathrm{~dB}$ edge taper and was designed to provide the beam clearance and optical element rims at $5 \omega$.

allows using the receiver cryostat in a steady position with the cryo-cooler and the receiver cartridges placed vertically, thus achieving a more compact cryostat design. A similar approach though with differently designed receiver cartridges was used for the ASTE telescope (Sugimoto et al. 2003). The SEPIA cryostat was built around a three-stage Sumitomo RDK-3ST cryo-cooler generation 6 , with $1 \mathrm{~W}$ at $4 \mathrm{~K}$ lift off power. In order to provide mechanical and cryogenic compatibility with the ALMA receiver cartridges, we used the same distances and dimensions of the cartridge holders as in the ALMA FE cryostat. Furthermore, the cartridge cooling thermal links at $110 \mathrm{~K}, 15 \mathrm{~K}$ and $4 \mathrm{~K}$ temperature stages have been produced by Rutherford Appleton Laboratory, UK to the ALMA FE specifications and used in the SEPIA cryostat. Figure 2, left shows the interior of the SEPIA cryostat with the mounted cartridge cooling thermal links and the Sumitomo cryo-cooler integrated into the cryostat. At the center of the cryostat, we used a stainless steel tube (protected by coaxial thermal shields) that connects the bottom and top bulkheads of the cryostat vacuum vessel and precludes the deflection of the bulkheads made off anodized aluminum.

The cryostat vacuum vessel is made of two aluminum bulkheads and a stainless steel tube interconnected using standard ISO-K claw clams. The top bulkhead has three ports to connect the vacuum pump, vacuum gauge, and the venting valve that are integrated with the SEPIA cryostat. All vacuum sealing is done with Viton ${ }^{\mathrm{TM}}$ O-rings. The SEPIA cryostat employs anti-vibration suspension of the cryo-cooler by using a bellow between the $300 \mathrm{~K}$ flange of the RDK-3ST cryo-cooler and the 


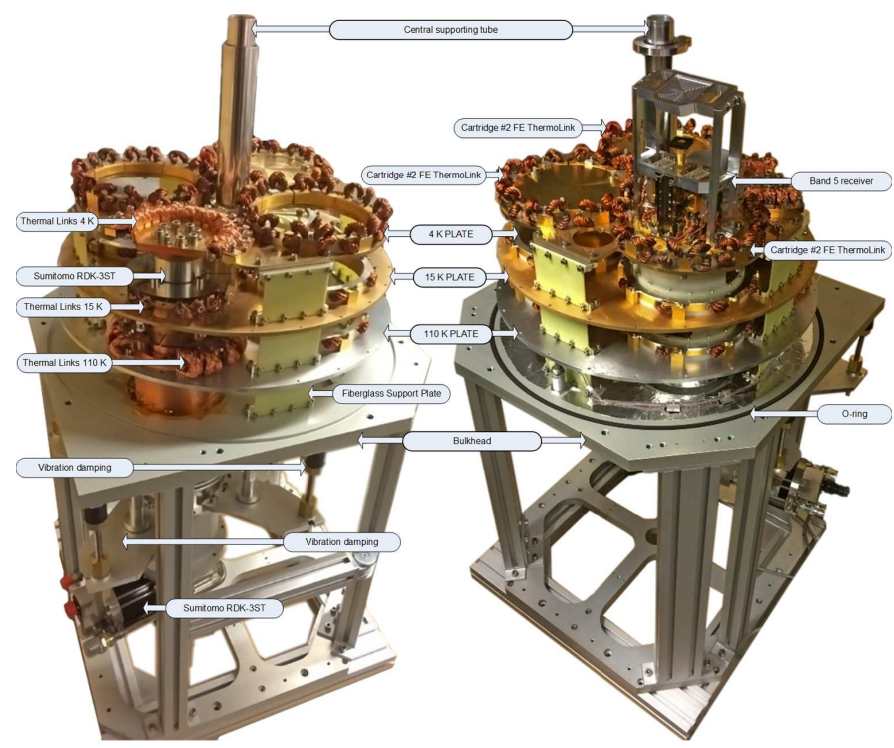

Fig. 2. SEPIA cryostat interior. Left: the cartridge cooling thermal links at $110 \mathrm{~K}, 15 \mathrm{~K}$ and $4 \mathrm{~K}$ temperature stages (purchased from RAL, UK) and the Sumitomo cryo-cooler integrated into the cryostat. All thermal connections are made via oxygen-free copper flexible braids to avoid vibration transfer. Below the bulkhead, the elements of the anti-vibration cold-head suspension system are shown. Right: SEPIA cryostat interior with ALMA Band 5 pre-production cartridge installed and the idle cartridge places blanked. Fiberglass support plates that also limit thermal conductivity flux sufficiently well providing the mechanical stability of the thermal decks.

cryostat to keep the vacuum with the vibration-damping rubber elements installed at the brackets providing the mechanical attachment of the cryo-cooler to the cryostat bottom bulkhead (Fig. 2, left). All thermal links between the cryo-cooler temperature stages and the elements of the receiver are made with flexible thermal contacts using braided $0.05 \mathrm{~mm}$ diameter oxygen-free copper wires with each thermal link having a cross-sectional area of about $6 \mathrm{~mm}^{2}$. This ensures that the vibration of the cryo-cooler is not transferred to the receiver, its supporting structure and the cryostat. The entire inner mechanical structure was simulated using Ansys ${ }^{\mathrm{TM}}$ FESS to verify that eigenfrequencies exceed $80 \mathrm{~Hz}$.

The total weight of the SEPIA receiver with tertiary optics and its supporting frame, integrated turbo-pump and with three receiver cartridges loaded is expected to be approximately $315 \mathrm{~kg}$. This also includes three cartridge loaders mounted at the receiver cartridge loading interface that could be flexibly arranged to fit any ALMA receiver cartridge. In order not to overload the APEX Cabin A receiver support structure, a weight-compensation scheme was implemented, comprising of the three supporting legs each equipped with the rubber foot and joint to the SEPIA cryostat via gas-spring. The gas springs each provide force at the level of $620 \pm 20 \mathrm{~N}$ giving a total of $1860 \mathrm{~N}$ of the weight compensation. Figure 3 shows a picture of the SEPIA receiver installed at its PI 2 position in the APEX Cabin A.

The cryostat temperature is monitored and controlled via a CryoCon ${ }^{\mathrm{TM}}$ cryogenic temperature controller, Model $24 \mathrm{C}$, which allows stabilizing the $4 \mathrm{~K}$ temperature deck to facilitate the SIS mixers gain stability and ensure that the sideband rejection performance of the SIS mixers is not affected by changing the physical temperature. Additionally, the CryoCon ${ }^{\mathrm{TM}}$ signal from the cryogenic temperature sensors is used by the receiver control system to interlock the receiver shut down at the events of a power

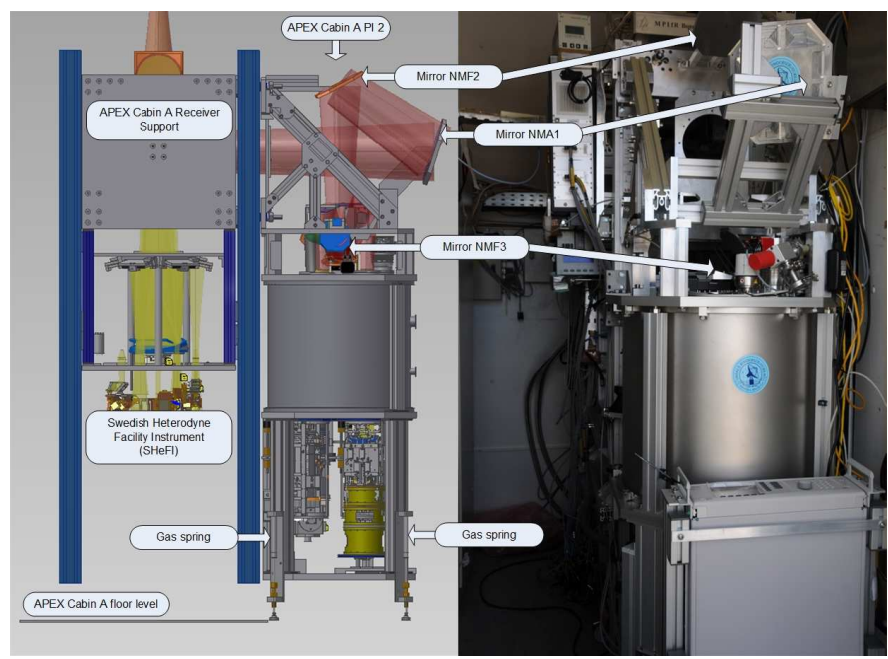

Fig. 3. SEPIA receiver. Left: CAD picture showing the entire assembly of the SEPIA cryostat and dedicated SEPIA tertiary optics attached to the APEX Cabin A instrument-supporting frame. The SEPIA support toward the Cabin A floor uses gas springs unloading the frame and providing stable mechanical contact with the cabin floor. Right: picture of the SEPIA receiver installed at its outer PI 2 position in the APEX Cabin A.

blackout, cold head or compressor failure when the raised temperature does not allow operating SIS mixers. A typical SEPIA cryostat cool-down time is around 8 hours reaching a physical temperature of about $3.9 \mathrm{~K}$ in the laboratory conditions and about $3.4 \mathrm{~K}$ at the telescope, respectively. The cryo-refrigerator capacity and SEPIA cryostat design allow operation of 3 cartridges without substantial changes in the physical temperature.

The SEPIA cryostat RF vacuum windows have the same design as in the ALMA FE cryostat, they are made of crystal quartz with Teflon coating and were produced by QMC Inc. (for SEPIA cartridge Bands 5 and 9). The SEPIA cryostat uses the same infrared (IR) filters as the corresponding bands of ALMA FE and those were delivered by ESO. The design of the SEPIA RF vacuum window holders and the IR filter brackets is such that it allows their exchange using a specialized tool without disassembling the cryostat, thus providing full flexibility of installing different frequency ALMA cartridges, that is, any of the ALMA Band 5-10 cartridges, on three positions of the SEPIA cryostat. The first channel pick-up mirror with its corresponding support bracket should be individually introduced for each of the (new) ALMA cartridges in order to accommodate them to the SEPIA tertiary optics.

\subsection{SEPIA receiver control and back end}

The SEPIA receiver control system hardware uses standard ALMA FE bias modules (FEBM) for each receiver cartridge, which communicate with the ALMA Monitoring and Control $(\mathrm{M} \& \mathrm{C})$ unit, all the hardware with its respective firmware was developed and built by NRAO. The M\&C unit communicates with the PC computer that is running the SEPIA control software via CANbus using a PCAN-USB interface from PEAK. All peripheral hardware communication with the control software is based on an Ethernet internal network and communication with the APEX control system (APECS; Muders 2017) is via a separate Ethernet connection. Figure A.1 shows the block diagram of the SEPIA receiver control system, which consists of the receiver itself together with all peripheral hardware. 
The SEPIA control system PC is a dual boot system. Engineering software running under MS Windows ${ }^{\mathrm{TM}} 7$ is based on IRTECON (Ermakov et al. 2001) and used for advanced tuning and diagnostic of the ALMA Band 5 receiver channel. For the ALMA Band 9 receiver channel, NOVA uses specialized scripting software to tune the receiver and check the hardware.

The observing control software is run under Linux and provided for all SEPIA channels by OSO. Tuning of the receivers is fully automatic and controlled by APECS via network communication based on a command language which is modeled after Standard Commands for Programmable Instruments (SCPI). In addition, the program offers a graphical user interface (GUI), allowing the observer to visually check the state of the frontend, inspect entries in the tuning table and perform manual tuning for testing purposes. The GUI also provides a plot of the typical atmospheric transmission at Chajnantor over the frequency band of the receiver, and marks current LO tuning and sideband locations. Furthermore, a log of all SCPI commands as well as a tabular view of raw CANbus readings are provided for debugging purposes.

At present, the Band 5 and Band 9 receivers are controlled via two independent programs, which share most of their code base, but differ in parameters and hardware addressing.

The SEPIA receiver uses the XFFTS spectrometer (Klein et al. 2012) provided as part of the APEX collaboration by MPIfR. The spectrometer has $4-8 \mathrm{GHz} \times 4$ bandwidth, which covers $100 \%$ of the SEPIA ALMA Band 5 receiver channel IF band and only $4-8 \mathrm{GHz} \times 2$ of the IF band of the SEPIA ALMA Band 9 receiver channel whereas the IF band $8-12 \mathrm{GHz}$ is not available in the current configuration. An upgrade of the IF system is planned for late 2018, which will cover 4-12 GHz IF bandwidth.

\subsection{SEPIA ALMA Band 5 receiver channel}

ALMA Band 5 receivers have been developed, designed and six pre-production cartridges have been built under EC FP 6 "Infrastructure Enhancement Program"1. The details of the receiver cartridge design and performance can be found in Billade et al. (2012). The produced pre-production ALMA Band 5 cartridges have been delivered to ALMA via ESO. When ALMA Band 5 full production started, it was decided to spare the pre-production cartridges and one of those was made available by ESO for the APEX SEPIA receiver. Several important modifications have been made by GARD to the pre-production ALMA Band 5 cartridge to bring it up-to-date. The most serious upgrade was done to the LO and WCA (Bryerton et al. 2013), the hardware version produced by NRAO for full ALMA Band 5 production was used with appropriate changes in the cartridge design.

The cartridge 2SB SIS mixer assemblies for both polarizations have been replaced by the latest version with modifications of the 2SB SIS mixers and IF hybrid assembly used for the full ALMA Band 5 production project, for example, a slightly extended RF band down to $158 \mathrm{GHz}$ that allows to avoid the "wings" of the $183 \mathrm{GHz}$ water line when observing with the upper sideband (Belitsky et al. 2018). The summary of the specifications for SEPIA ALMA Band 5 receiver channel is presented in Table 1. In Fig. 4, the picture of the modified ALMA Band 5 pre-production cartridge is shown along with the noise temperature and sideband rejection ratio performance for both polarizations performed in the lab keeping the SIS mixers at

1 ALMA Band 5, EC Framework Program 6 (FP6), under infrastructure enhancement, Contract 515906.
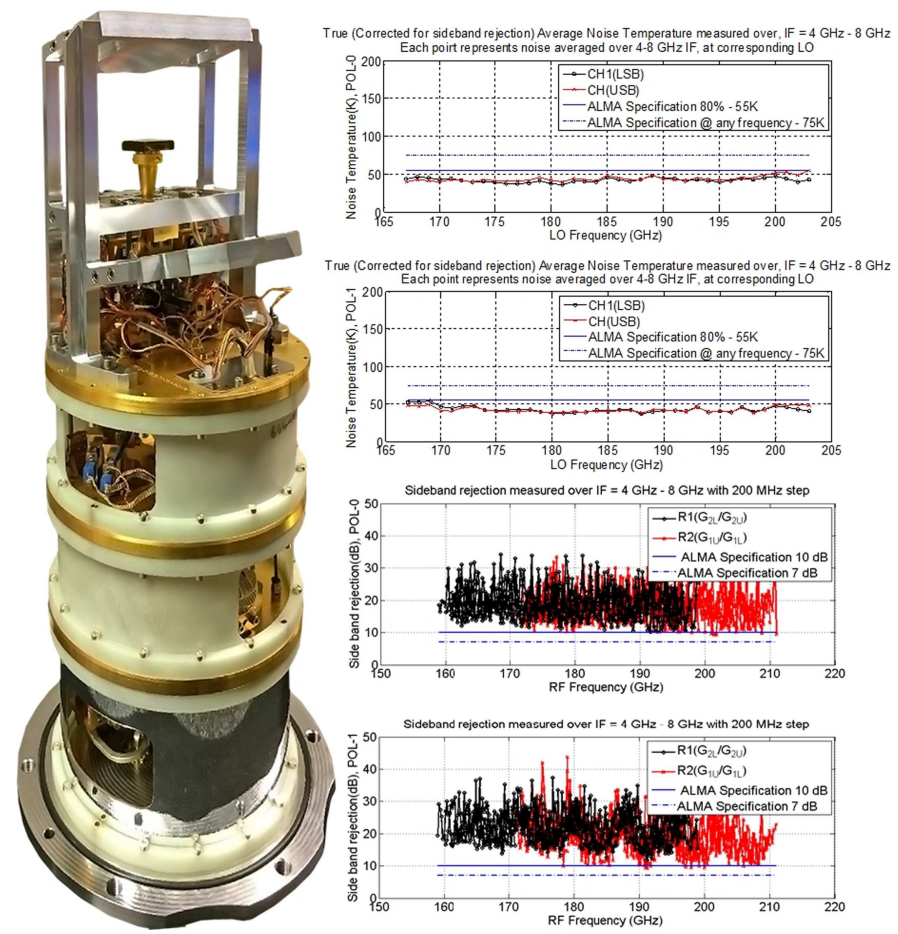

Fig. 4. Left: SEPIA ALMA Band 5 pre-production receiver cartridge. Right: measured noise temperatures and the sideband rejection ratio of the SEPIA Band 5 receiver cartridge.

$4 \mathrm{~K}$ physical temperature. As mentioned above, the SEPIA cryostat is equipped with an active temperature stabilization system, which allows stabilizing of the SIS mixer physical temperature down to milli-Kelvin level. This ensures that the optimum 2SB mixer tuning setting for the best noise temperature and the sideband-rejection will remain stable with changes of the cold-head physical temperature which may occur, for example, between the cold head service periods.

Technical commissioning of the SEPIA receiver with the ALMA Band 5 receiver band installed was performed in February-March 2015. The commissioning included hardware installation, for example, reference LO PLL synthesizer, IF switches, vacuum control gauge, turbo-pump, computerized control system, etc., putting in place and alignment of the SEPIA tertiary optics and installing the SEPIA cryostat and the cold head compressor. The entire system was tested for integrity and the SEPIA Band 5 beam alignment was verified using small size nitrogen-cooled absorbers at all tertiary optics components and at the APEX Cabin $\mathrm{C}$ at the antenna focal plane.

\subsection{SEPIA ALMA Band 9 receiver channel}

The NOVA instrumentation group at the Kapteyn Astronomical Institute in Groningen, The Netherlands, already produced a full set of ALMA Band 9 cartridges. For SEPIA, the NOVA group built another Band 9 cartridge at ALMA specifications and delivered it to the SEPIA PIs, ESO and OSO. This SEPIA ALMA Band 9 receiver has DSB dual polarization SIS mixers operating in the RF band of 599.77-722.15 GHz. The summary of the specifications for SEPIA ALMA Band 9 receiver channel is presented in Table 2. The details on technology and performance of the ALMA Band 9 cartridge can be found in Baryshev et al. (2015). The ALMA Band 9 LO, WCA and FE bias module were produced by NRAO and jointly purchased by ESO and 
Table 1. Summary of the SEPIA ALMA Band 5 specifications and performance.

\begin{tabular}{lll}
\hline \hline Item & Specifications & Comment \\
\hline Technology & 2SB SIS mixers, dual polarization & \\
RF band & $157.36-211.64 \mathrm{GHz}$ & \\
IF band & $4-8 \mathrm{GHz}$ & For each sideband \\
IF bandwidth & $4 \times 4 \mathrm{GHz}$ & \\
SSB noise temperature & $<50 \mathrm{~K}$ & Averaged over RF band $<45 \mathrm{~K}$ \\
Sideband rejection ratio & $>10 \mathrm{~dB}$ & Averaged over IF $>16 \mathrm{~dB}$ \\
Cross-polarization & $<-23 \mathrm{~dB}$ & At the cartridge RF window \\
\hline
\end{tabular}
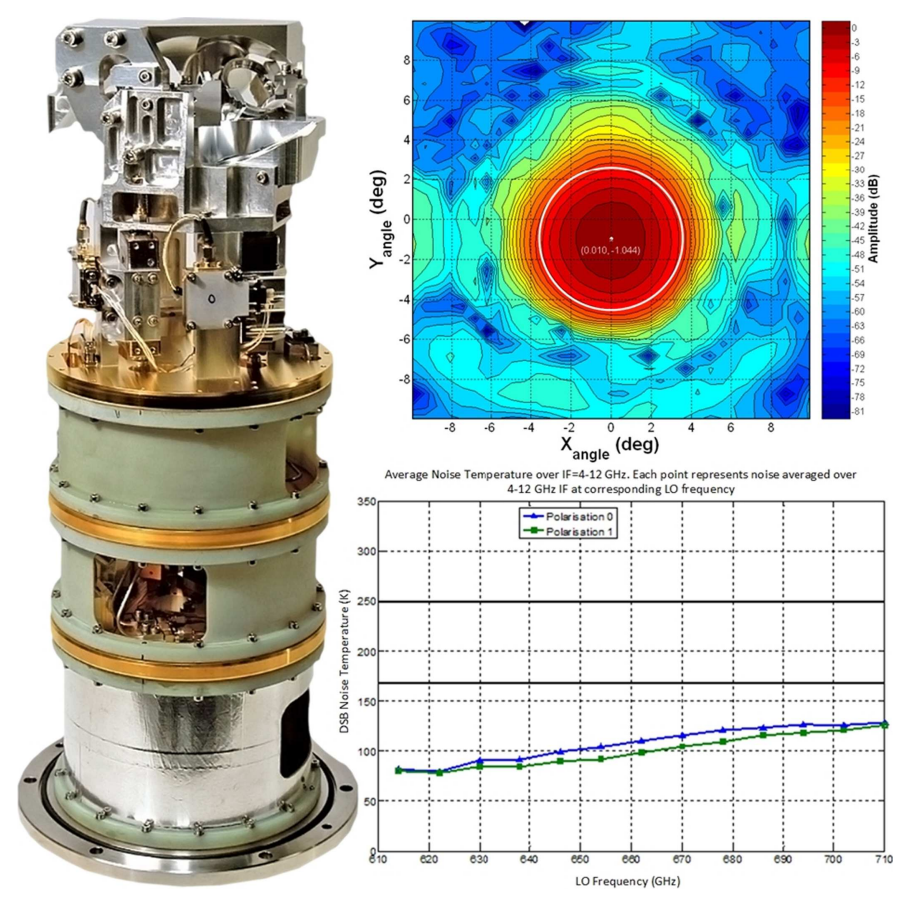

Fig. 5. Left: SEPIA ALMA Band 9 pre-production receiver cartridge. Top right: typical co-polar beam pattern in a Band 9 cartridge (polarization 0 in cartridge \#63, picture from Baryshev et al. (2015), with the secondary telescope mirror indicated (white circle). Bottom right: noise temperatures of the SEPIA Band 9 cartridge.

OSO for the SEPIA project. Figure 5 shows the picture of the ALMA Band 9 production cartridge and the noise temperature for both polarizations with the measurements performed in the lab keeping the SIS mixers at $4 \mathrm{~K}$ physical temperature for the ALMA Band 9 Cartridge \#74 installed in SEPIA.

Technical commissioning of the SEPIA ALMA Band 9 receiver channel was performed in February-March 2016 by a joint NOVA-GARD team. The commissioning included hardware installation, IF switches, computerized control system extension and software update. The entire system was tested for integrity and the SEPIA Band 9 beam alignment was verified using small size nitrogen-cooled absorbers at all relay mirrors and finally at the APEX Cabin $\mathrm{C}$ at the antenna focal plane.

The current plan is to replace the SEPIA ALMA Band 9 DSB receiver channel in February-March 2018 with a new receiver cartridge, which employs 2SB SIS mixers with four IF outputs, that is, 4-12 GHz USB and LSB for each polarization, and otherwise has the same hardware and layout as the ALMA Band 9 cartridge.

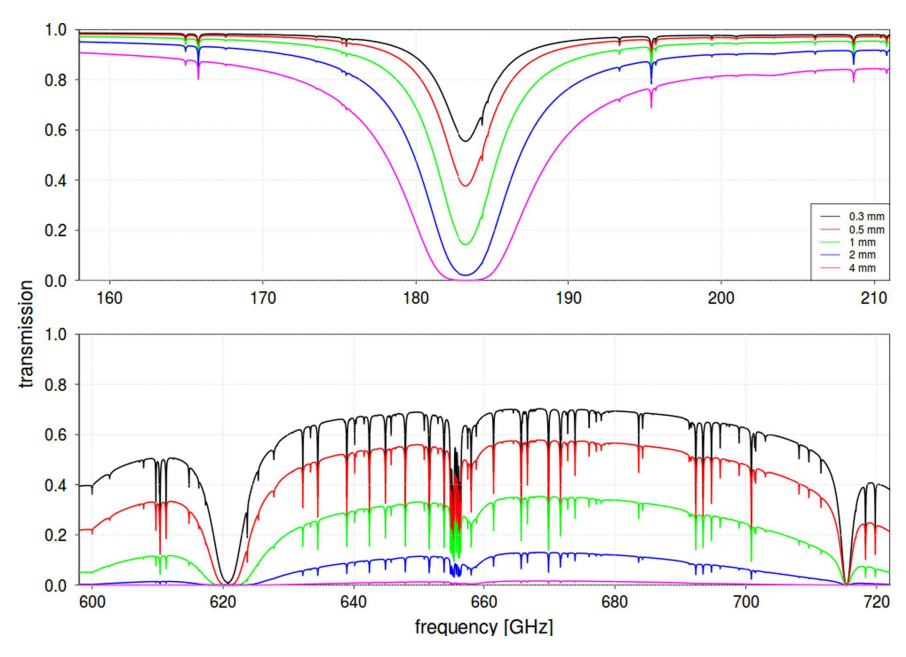

Fig. 6. Atmospheric transmission at Llano de Chajnantor (Paine 2017) for the frequency ranges of the Band 5 (upper panel) and Band 9 (lower panel) receivers.

\section{Sky commissioning}

The sky commissioning of the SEPIA Band 5 receiver was successfully performed between February and September 2015. The sky commissioning of Band 9 has been started in June 2016. The atmospheric transmission at Llano de Chajnantor in the frequency range of the SEPIA Band 5 and Band 9 receivers is shown in Fig. 6. A number of on-sky tests have to be conducted before a new instrument can be offered for observing time. Tuning Band 5 every $0.5 \mathrm{GHz}$, we confirmed that the whole tuning range from $157.36-211.64 \mathrm{GHz}$ is accessible. Observing strong water masers as part of the science verification, Humphreys et al. (2017) determined the sideband suppression level to $17.7 \mathrm{~dB}$ from comparing the water line in the upper sideband to its ghost line in the lower sideband.

To determine the beam coupling efficiencies for Band 5, we observed Mars on UT 2016 April 6 and 7 at 208 GHz, and Jupiter on UT 2016 August 11 at $170 \mathrm{GHz}$. The respective apparent sizes of these planets were 12.7" for Mars and 31.4" for Jupiter. From the same planet observations, we derive deconvolved beam sizes of about 28.6" at $208 \mathrm{GHz}$ and $31.7^{\prime \prime}$ at $170 \mathrm{GHz}$. This means the apparent size of Jupiter matched the beam size, while Mars was an unresolved source (semi-extended in the Herschel/HIFI nomenclature). Assuming an antenna far-field forward efficiency $\eta_{f}=0.95$ (from Güsten et al. 2006, with $10 \%$ uncertainties across the 160-211 GHz range), we find beam coupling efficiencies of $0.67 \pm 0.05$ for Jupiter and $0.72 \pm 0.07$ for Mars, corresponding to $\mathrm{Jy} \mathrm{K}^{-1}$ factors of $33.8 \pm 2.5$ and $38.4 \pm 2.8$ for resolved and unresolved sources, respectively. 
Table 2. Summary of the SEPIA ALMA Band 9 specifications and performance.

\begin{tabular}{lll}
\hline \hline Item & Specifications & Comment \\
\hline Technology & DSB SIS mixers, dual polarization & \\
RF band & $599.77-722.15 \mathrm{GHz}$ & \\
IF band & $4-12 \mathrm{GHz}$ & DSB operation \\
IF bandwidth & $2 \times 8 \mathrm{GHz}$ & Currently limited by the FFTS to $2 \times 4 \mathrm{GHz}$ \\
DSB noise temperature & $75-125 \mathrm{~K}$ & Averaged over RF band $\approx 100 \mathrm{~K}$ \\
Cross-polarization & $-15.5 \mathrm{~dB}$ & At the cartridge RF window \\
\hline
\end{tabular}

To determine the beam coupling efficiencies for Band 9, we observed Jupiter on UT 2017 April 22, and Uranus on UT 2017 May 21, both at $691.5 \mathrm{GHz}$. The apparent sizes for Uranus and Jupiter were $3.4^{\prime \prime}$ and 42.6", respectively. From observations toward Callisto, which has an apparent size of $1.5^{\prime \prime}$, we determine a deconvolved beam size of $8.8 \pm 0.5^{\prime \prime}$ at $691.5 \mathrm{GHz}$. Assuming the same antenna far-field forward efficiency $\eta_{\mathrm{f}}=0.95$ as above, we find beam coupling efficiencies of $0.46 \pm 0.02$ for Jupiter and $0.37 \pm 0.03$ for Uranus, corresponding to $\mathrm{Jy} \mathrm{K}^{-1}$ factors of $63 \pm$ 3 and $79 \pm 6$, for resolved and unresolved sources, respectively.

These values were obtained after careful optimization of the antenna performance (replacement of the secondary surface and dish setting following holography results). At these high frequencies, the effective performance depends critically on both the antenna and the instrument. While we expect the performance of the instrument to be relatively stable, the antenna efficiencies can vary on time scales of weeks or months, due to the external weather factors. Users are encouraged to perform efficiency measurements as part of their science projects, or to contact APEX staff for the most recent values to be used.

Pointing and focus observations at APEX are conducted toward compact sources with either strong continuum or line emission. For the other APEX heterodyne instruments, these observations are often done in the main transitions of the $\mathrm{CO}$ molecule. Since the Band 5 frequency range does not contain a $\mathrm{CO}$ transition, we had to find sources that strongly emit in other molecular transitions. We chose evolved stars that are bright in $\mathrm{HCN}(2-1)$ or $\mathrm{SiO}(4-3) v[0-3]$ masers. After testing their quality as pointing sources, we constructed a pointing catalog, which covers the whole LST range (Fig. 7, Table B.1). The pointing sources are being used to compile a good pointing model for Band 5, using the professional version of the Telescope Pointing Analysis System TPOINT ${ }^{2}$ (Wallace 1994). With this receiver, pointing accuracies better than $2.5^{\prime \prime}$ are achieved.

The receiver stability was tested using a series of Allan variance measurements, both in spectral and total power mode. Results are shown in Fig. 8. For all measurements, a channel resolution of $1.2 \mathrm{MHz}$ was used. For both Band 5 and Band 9 spectroscopic stability times are always well above a few times $100 \mathrm{~s}$. For the quoted resolution, even the total power stability is typically above $100 \mathrm{~s}$, or above $30 \mathrm{~s}$ when scaled to $10 \mathrm{MHz}$ resolution.

\section{Calibration of Band 5 data}

The online calibration of spectral line observations at the APEX telescope is based on SKY-HOT-COLD calibration scans immediately before the spectral line data is taken. During these scans, observations of the blank sky, a hot load at ambient

2 http://www.tpointsw.uk

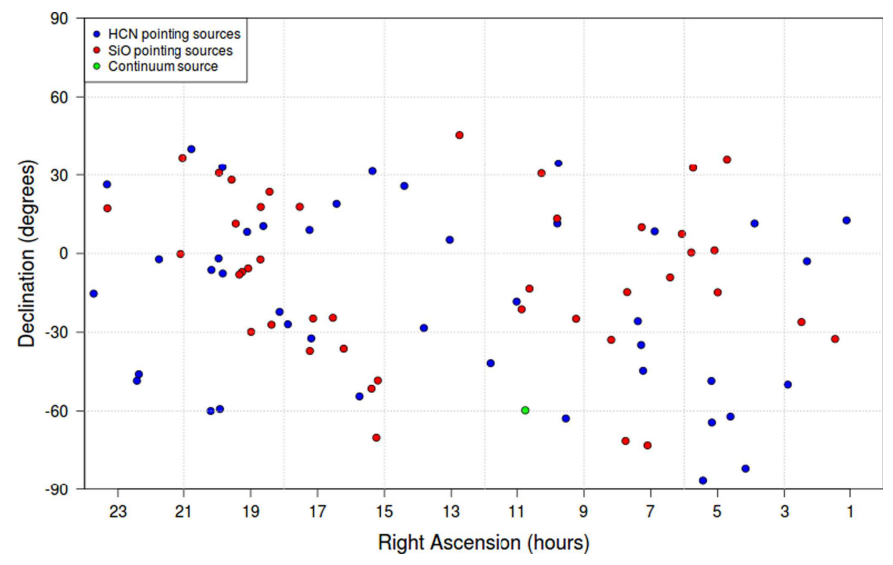

Fig. 7. Sky distribution of SEPIA Band 5 pointing sources (see Table B.1).

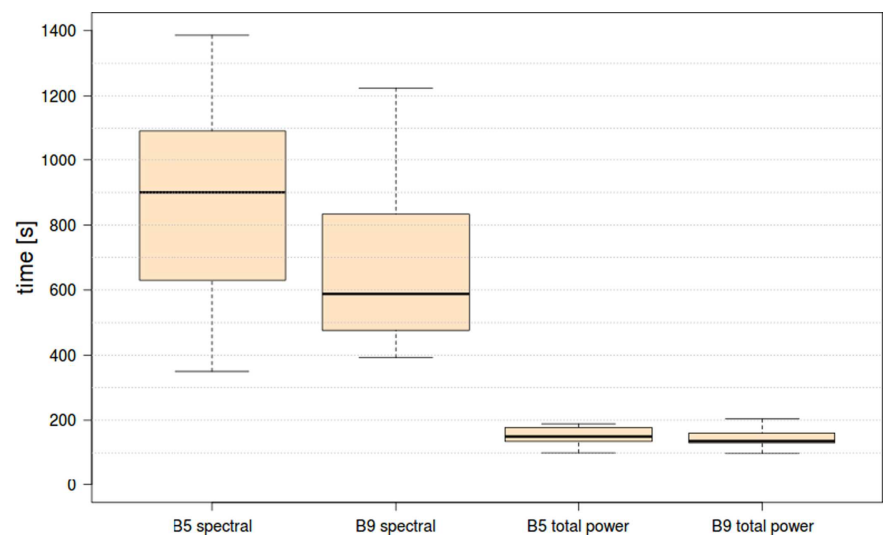

Fig. 8. Results from Allan variance measurements. The bold horizontal lines mark the median results of all measurements taken, the top and bottom of the boxes show the 25 th and 75 th percentile, i.e., outline the middle $50 \%$ of the data.

temperature and a cold load at liquid nitrogen temperature are conducted. From the HOT and COLD signal, the Online Calibrator determines the receiver temperature. From the SKY phase, it calculates the sky temperature, correcting for spillover and forward efficiency. Based on the elevation of the SKY observations and a sophisticated atmospheric model, ATM (Pardo et al. 2001), the opacity in image and signal band is determined. In the online calibration, an average opacity is calculated based on ten equally spaced frequencies across the $2.5 \mathrm{GHz}$ band pass. This is sufficient if the atmosphere is reasonably flat. However, in Band 5 due to the water absorption of the atmosphere, this approach is insufficient for observations close to $183.3 \mathrm{GHz}$. This type of data needs to be recalibrated offline at the telescope. In the offline calibration, opacity values are determined 
Table 3. Astrophysically important molecular transitions within the frequency coverage of SEPIA Band 5.

\begin{tabular}{|c|c|c|c|}
\hline Molecule & Transition & Frequency $(\mathrm{GHz})$ & Comment \\
\hline $\mathrm{CH}_{3} \mathrm{CN}$ & $J=9-8$ & 165.60 & Several $K$-components \\
\hline $\mathrm{H}_{2} \mathrm{~S}$ & $J_{K a, K c}=1_{1,1}-1_{0,1}$ & 168.76 & \\
\hline $\mathrm{HC}^{18} \mathrm{O}^{+}$ & $J=2-1$ & 170.32 & \\
\hline $\mathrm{CH}_{3} \mathrm{CCH}$ & $J=10-9$ & 170.90 & Several $K$-components \\
\hline $\mathrm{SiO}, v=2$ & $J=4-3$ & 171.28 & \\
\hline $\mathrm{HC}^{15} \mathrm{~N}$ & $J=2-1$ & 172.11 & \\
\hline $\mathrm{SiO}, v=1$ & $J=4-3$ & 172.48 & \\
\hline $\mathrm{H}^{13} \mathrm{CN}$ & $J=2-1$ & 172.68 & \\
\hline $\mathrm{H}^{13} \mathrm{CO}^{+}$ & $J=2-1$ & 173.51 & \\
\hline $\mathrm{SiO}, v=0$ & $J=4-3$ & 173.69 & \\
\hline $\mathrm{CCH}$ & $N=2-1$ & 174.70 & Several lines \\
\hline $\mathrm{HCN}$ & $J=2-1$ & 177.26 & \\
\hline $\mathrm{HCO}^{+}$ & $J=2-1$ & 178.38 & \\
\hline $\mathrm{HNC}$ & $J=2-1$ & 181.32 & \\
\hline $\mathrm{H}_{2} \mathrm{O}$ & $J_{K a, K c}=3_{1,3}-2_{2,0}$ & 183.31 & \\
\hline $\mathrm{CH}_{3} \mathrm{CN}$ & $J=10-9$ & 183.90 & Several $K$-components \\
\hline${ }^{13} \mathrm{CS}$ & $J=4-3$ & 184.98 & \\
\hline $\mathrm{N}_{2} \mathrm{H}^{+}$ & $J=2-1$ & 186.34 & \\
\hline $\mathrm{CH}_{3} \mathrm{CCH}$ & $J=11-10$ & 187.90 & Several $K$-components \\
\hline $\mathrm{C}^{34} \mathrm{~S}$ & $J=4-3$ & 192.82 & \\
\hline $\mathrm{CH}_{3} \mathrm{OH}$ & $J=4-3$ & 193.50 & Several lines \\
\hline CS & $J=4-3$ & 195.95 & \\
\hline $\mathrm{CH}_{3} \mathrm{CN}$ & $J=11-10$ & 202.30 & Several $K$-components \\
\hline $\mathrm{H}_{2}^{18} \mathrm{O}$ & $J_{K a, K c}=3_{1,3}-2_{2,0}$ & 203.41 & \\
\hline $\mathrm{CH}_{3} \mathrm{CCH}$ & $J=12-11$ & 205.00 & Several $K$-components \\
\hline
\end{tabular}

in chunks of 128 channels (the resolution of the atmospheric model). The online calibrated data underestimates the emission around $183 \mathrm{GHz}$ by up to $30 \%$ (e.g., Immer et al. 2016). The goal is to upgrade the online calibration to provide such optimally calibrated data straight away.

\section{Science with SEPIA Band 5}

After commissioning and science verification, over 100 regular proposals have been accepted on SEPIA in the first two years of operations. The SEPIA proposals represent between $25 \%$ and $50 \%$ of the accepted proposals in the past four observing periods, and over 1000 hours have already been observed. Seven science papers have been published at the time of writing, and a science meeting describing these results was held at ESO (De Breuck et al. 2017) ${ }^{3}$.

One of the main science drivers for an ALMA Band 5 receiver at APEX was the possibility to observe the $183.3 \mathrm{GHz}$ water line. Interstellar water emission was first detected by (Cheung et al. 1969) and since then, the Herschel Space Observatory has shown that gaseous water is widespread in molecular clouds in our Galaxy (van Dishoeck et al. 2011). With APEX, surveys for both maser as well as thermal water emission toward a large number of sources are feasible. The water molecule is an important tracer of the energetic processes which take place during the formation of low- and high-mass stars. In combination with the lower optical depth $\mathrm{H}_{2}^{18} \mathrm{O}$ transition at $203.4 \mathrm{GHz}$, water observations probe the water content even in the inner parts of

\footnotetext{
3 See also https://www.eso.org/sci/meetings/2017/band5/ program.html
}

hot molecular cores, complementing space-based water observations (e.g., from Herschel). The $183.3 \mathrm{GHz}$ line has also been detected in the O-rich circumstellar envelopes of several evolved stars with mass-loss rates exceeding $10^{-6} M_{\odot} \mathrm{yr}^{-1}$ (GonzalezAlfonso et al. 1998; González-Alfonso \& Cernicharo 1999). Observations of this transition with APEX will thus allow the study of the water abundance in evolved stars. In addition, the degree of the linear polarization of the $183.3 \mathrm{GHz}$ water maser emission can be determined from dual polarization observations with Band 5 (Humphreys et al. 2017). Furthermore, water maser observations will provide information about the water content of nearby galaxies $(z<0.1)$ as well as the high temperature and density regions of active galactic nuclei (AGNs). In the Seyfert 2 galaxy $\mathrm{NGC} 4945,183.3 \mathrm{GHz} \mathrm{H}_{2} \mathrm{O}$ megamaser emission was detected for the first time with SEPIA Band 5 (Humphreys et al. 2016). It is the strongest extragalactic submillimeter water maser detected to date. The emission seems to be dominated by that from the AGN central engine.

Galametz et al. (2016) recently detected water $(183.3 \mathrm{GHz})$ and the methanol $(4-3)$ group $(193.5 \mathrm{GHz})$ in the ultra-luminous infrared galaxy Arp 220. No time variations are observed in the megamaser water line compared to previous observations. This supports arguments against an AGN nuclear origin for the line. The $J=2-1$ transitions of the nitrogen-bearing species $\mathrm{HCN}$, $\mathrm{HNC}$, and $\mathrm{N}_{2} \mathrm{H}^{+}$as well as $\mathrm{HCO}^{+}$are available in the SEPIA Band 5 frequency range. Their isotopologues $\mathrm{H}^{13} \mathrm{CN}, \mathrm{HC}^{15} \mathrm{~N}$, $\mathrm{H}^{13} \mathrm{CO}^{+}$, and $\mathrm{HC}^{18} \mathrm{O}^{+}$are observable in one frequency setting as are $\mathrm{HCN}$ and $\mathrm{HCO}^{+}$. These transitions serve as low-energy complements to the higher J-lines which are covered by other APEX bands, probing material that is colder and of lower density. In evolved stars, simultaneous observations of $\mathrm{HCN}$ and $\mathrm{SiO}$ allow the determination of the $\mathrm{HCN}$ to $\mathrm{SiO}$ intensity ratio $(\mathrm{HCN} / \mathrm{SiO})$ 
Table 4. Astrophysically important molecular transitions within the frequency coverage of SEPIA Band 9.

\begin{tabular}{|c|c|c|c|}
\hline Molecule & Transition & Frequency (GHz) & Comment \\
\hline $\mathrm{H}^{13} \mathrm{CO}+$ & $J=7-6$ & 607.18 & \\
\hline $\mathrm{SiO}$ & $J=14-13$ & 607.61 & \\
\hline $\mathrm{HN}^{13} \mathrm{C}$ & $J=7-6$ & 609.51 & \\
\hline $\mathrm{HCN}-\mathrm{v} 2$ & $J=7-6$ & 623.36 & \\
\hline $\mathrm{HCO}^{+}$ & $J=7-6$ & 624.21 & \\
\hline $\mathrm{SiH}$ & ${ }^{2} \Pi F_{1} ; J=3 / 2^{+}-J=1 / 2^{+}$ & 624.92 & Several lines \\
\hline $\mathrm{H}^{37} \mathrm{Cl}$ & $J=1-0$ & 624.96 & Several lines \\
\hline $\mathrm{H}^{35} \mathrm{Cl}$ & $J=1-0$ & 625.90 & Several lines \\
\hline $\mathrm{C}^{34} \mathrm{~S}$ & $J=13-12$ & 626.34 & \\
\hline $\mathrm{HNC}$ & $J=7-6$ & 634.51 & \\
\hline${ }^{30} \mathrm{SiO}$ & $J=15-14$ & 635.22 & \\
\hline $\mathrm{CS}$ & $J=13-12$ & 636.53 & \\
\hline${ }^{29} \mathrm{SiO}$ & $J=15-14$ & 642.80 & \\
\hline $\mathrm{SiO}$ & $J=15-14$ & 650.96 & \\
\hline $\mathrm{DCN}$ & $J=9-8$ & 651.57 & \\
\hline $\mathrm{C}^{18} \mathrm{O}$ & $J=6-5$ & 658.55 & \\
\hline${ }^{13} \mathrm{CO}$ & $J=6-5$ & 661.07 & \\
\hline $\mathrm{C}^{17} \mathrm{O}$ & $J=6-5$ & 674.01 & \\
\hline${ }^{30} \mathrm{SiO}$ & $J=16-15$ & 677.51 & \\
\hline $\mathrm{HCS}^{+}$ & $J=16-15$ & 682.44 & \\
\hline $\mathrm{CS}$ & $J=14-13$ & 685.43 & \\
\hline${ }^{29} \mathrm{SiO}$ & $J=16-15$ & 685.59 & \\
\hline $\mathrm{HC}^{15} \mathrm{~N}$ & $J=8-7$ & 688.28 & \\
\hline $\mathrm{H}^{13} \mathrm{CN}$ & $J=8-7$ & 690.55 & \\
\hline $\mathrm{CO}$ & $J=6-5$ & 691.47 & \\
\hline $\mathrm{H}^{13} \mathrm{CO}^{+}$ & $J=8-7$ & 693.88 & \\
\hline $\mathrm{SiO}$ & $J=16-15$ & 694.29 & \\
\hline $\mathrm{HN}^{13} \mathrm{C}$ & $J=8-7$ & 696.53 & \\
\hline HCN-v2 & $J=8-7$ & 708.79 & Several lines \\
\hline $\mathrm{HCN}$ & $J=8-7$ & 708.88 & \\
\hline $\mathrm{HCO}^{+}$ & $J=8-7$ & 713.34 & \\
\hline${ }^{30} \mathrm{SiO}$ & $J=17-16$ & 719.79 & \\
\hline$C^{34} S$ & $J=15-14$ & 722.58 & \\
\hline
\end{tabular}

which is indicative of the star's chemical type (Olofsson et al. 1998).

The symmetric top molecules $\mathrm{CH}_{3} \mathrm{CN}$ and $\mathrm{CH}_{3} \mathrm{CCH}$ have several K-ladders in the band. Also, the methanol $J=4-3$ line forest around $193.5 \mathrm{GHz}$ and the set of lines of $\mathrm{CCH}(2-1)$ around 174.7 GHz are accessible with SEPIA Band 5. All these transitions are excellent temperature tracers of the denser molecular gas. They are more sensitive to lower temperatures than the corresponding transitions in other APEX bands. Molinari et al. (2016) observed $\mathrm{CH}_{3} \mathrm{CCH}$ (12-11) toward 51 dense clumps and determined their gas temperatures. Many other molecules have transitions in the SEPIA Band 5 frequency range. Table 3 lists some of the astrophysically important ones. A corresponding list for SEPIA Band 9 is given in Table 4.

For all sources at $z<0.615$, at least one $\mathrm{CO}$ transition falls within the frequency range of the SEPIA Band 5 receiver. This allows observations of high-J $\mathrm{CO}$ transitions in bright submillimeter galaxies to investigate their $\mathrm{CO}$ spectral line energy distributions or confirm their redshifts. Strandet et al. (2016), who studied the redshift distribution of gravitationally lensed dusty star-forming galaxies discovered from the South Pole Telescope, used the SEPIA CO observations of two sources to confirm their redshifts. Detecting [CII] in highly redshifted sources $(z<8.9)$ is challenging for APEX but extremely rewarding.

Due to the $8 \mathrm{GHz}$ bandwidth per polarization channel, the whole frequency range of SEPIA Band 5 can be covered with only eight frequency tunings with several $100 \mathrm{MHz}$ overlap. As ESO science verification project, Immer et al. (in prep.) observed the frequency range $159.2-210.7 \mathrm{GHz}$ toward the high-mass star forming complex Sgr B2, proving once again the line richness of this source and illustrating the large number of transitions that can be detected in SEPIA Band 5.

\section{Conclusion}

In this paper, we describe the APEX SEPIA receiver which was installed in APEX Cabin A at the beginning of 2015. The SEPIA receiver extensively uses ALMA technology developments and is a flexible platform, which allows the use of up to three regular ALMA receiver cartridges and new receivers. Currently, SEPIA provides APEX observers with two stateof-the-art dual-polarization receivers, covering two frequency channels 158-211 GHz, ALMA Band 5, and 600-720 GHz, ALMA Band 9. The excellent performance of the receiver was confirmed during science commissioning. Besides its advanced 
latest technology and current capabilities, SEPIA provides an excellent opportunity of testing and verifying experimental receivers for future ALMA upgrades, for example, planned in 2018 an upgrade of SEPIA ALMA Band 9 receiver channel with 2SB SIS mixers. We expect that coming upgrades will further improve the receiver and give greater opportunities for observing with APEX. All current SEPIA receiver channels are available to all APEX observers, and all data are publicly available after a one-year proprietary period.

Acknowledgements. We would like to thank the APEX science team for valuable assistance during on-sky commissioning of the new receiver. Prof. K. Menten, MPIfR, is acknowledged for making available the XFFTS spectrometer for use with SEPIA. We would like to acknowledge Kamaljeet S. Saini and NRAO for providing the LO and Band 5 and Band 9 warm cartridge hardware. We also thank Karina Celedón and Alicia Garafulich for their help with the logistics.

\section{References}

Baryshev, A. M., Hesper, R., Mena, F. P., et al. 2015, A\&A, 577, A129

Belitsky, V., Bylund, M., Desmaris, V., 2018, A\&A, 611, A98

Billade, B., Nyström, O., Meledin, D., et al. 2012, IEEE Trans. Terahertz Sci. Technol., 2, 208

Bryerton, E., Saini, K., Muehlberg, J., Vaselaar, D., \& Thacker, D. 2013, IEEE MTT-S Int. Microw. Symp. Dig., 6697622

Cheung, A. C., Rank, D. M., Townes, C. H., Thornton, D. D., \& Welch, W. J. 1969, Nature, 221, 626
De Breuck, C., Testi, L., \& Immer, K. 2017, The Messenger, 167, 11

Ermakov, A. B., Shitov, S. V., Baryshev, A. M., Koshelets, V. P., \& Luinge, W. 2001, IEEE Trans. Appl. Supercond, 11, 840

Galametz, M., Zhang, Z.-Y., Immer, K., et al. 2016, MNRAS, 462, L36

González-Alfonso, E. \& Cernicharo, J. 1999, ApJ, 525, 845

Gonzalez-Alfonso, E., Cernicharo, J., Alcolea, J., \& Orlandi, M. A. 1998, A\&A, 334, 1016

Güsten, R., Nyman, L. Å., Schilke, P., et al. 2006, A\&A, 454, L13

Humphreys, E. M. L., Vlemmings, W. H. T., Impellizzeri, C. M. V., et al. 2016, A\&A, 592, L13

Humphreys, E. M. L., Immer, K., Gray, M. D., et al. 2017, A\&A, 603, A77

Immer, K., Belitsky, V., Olberg, M., et al. 2016, The Messenger, 165, 13

Klein, B., Hochgürtel, S., Krämer, I., et al. 2012, A\&A, 542, L3

Laing, R., Maiolino, R., Rykaczewski, H., \& Testi, L. 2010, The Messenger, 141, 41

Molinari, S., Merello, M., Elia, D., et al. 2016, ApJ, 826, L8

Muders, D. 2017, APECS User Manual, Tech. Rep. APEX-MPI-MAN-0011

Olofsson, H., Lindqvist, M., Nyman, L.-A., \& Winnberg, A. 1998, A\&A, 329, 1059

Paine, S. 2017, The am atmospheric model (v. 9.2)

Pardo, J. R., Cernicharo, J., \& Serabyn, E. 2001, IEEE Trans. Antennas Propag., 49, 1683

Strandet, M. L., Weiss, A., Vieira, J. D., et al. 2016, ApJ, 822, 80

Sugimoto, M., Sekimoto, Y., Yokogawa, S., et al. 2003, in Fourteenth International Symposium on Space Terahertz Technology, ed. C. Walker \& J. Payne, 535

van Dishoeck, E. F., Kristensen, L. E., Benz, A. O., et al. 2011, PASP, 123, 138

Wallace, P. T. 1994, Starlink User Note, 100 


\section{Appendix A: Block diagram}

\section{SEPIA Control Sys tem overview}

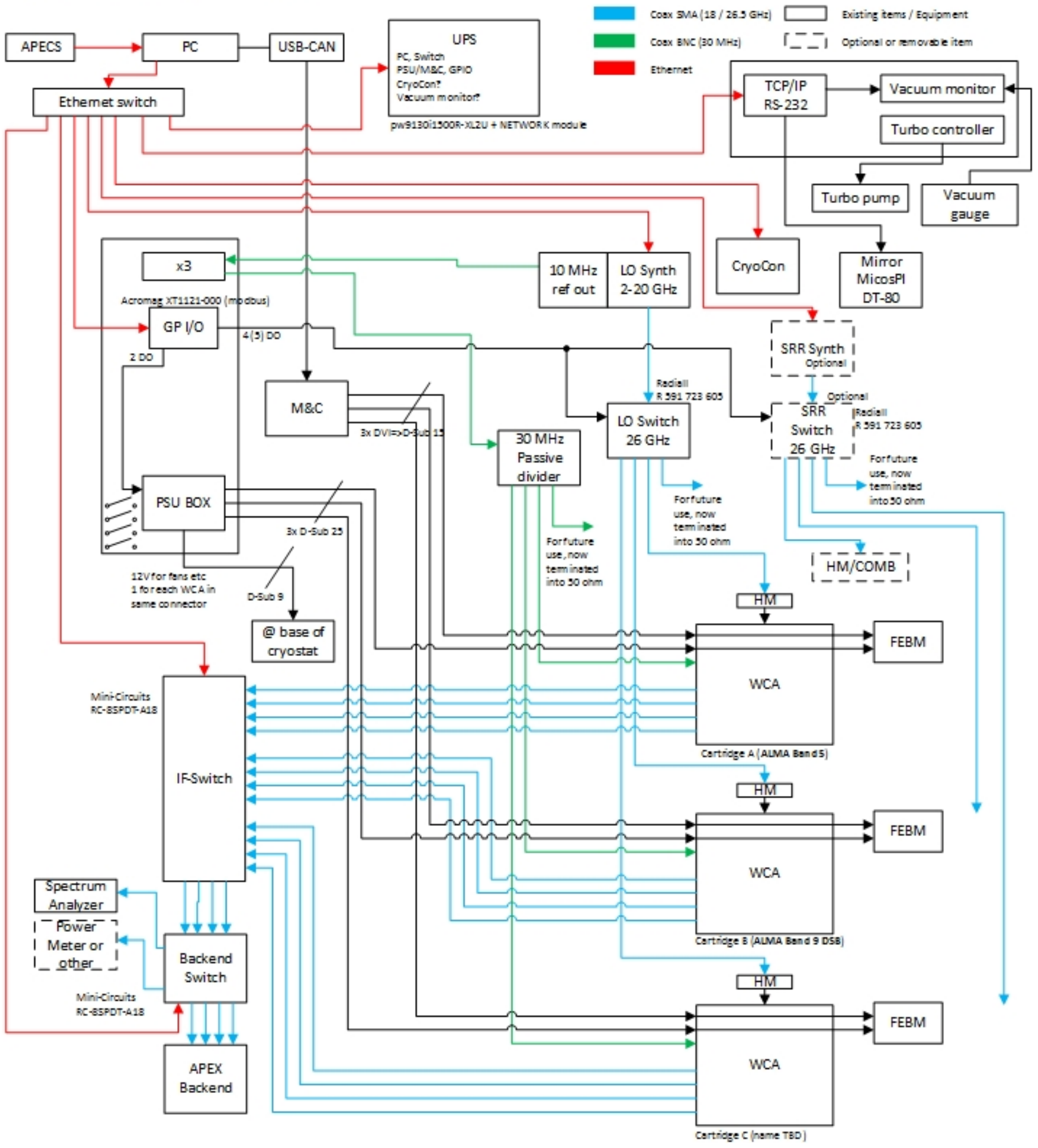

Fig. A.1. Block diagram of the SEPIA receiver control system with all peripheral hardware. 
Appendix B: Pointing sources

Table B.1. Coordinates of the SEPIA Band 5 pointing sources, obtained from different catalogs.

\begin{tabular}{|c|c|c|c|c|}
\hline Name & RA [hms] & $\operatorname{Dec}\left[{ }^{0}, \prime \prime\right]$ & Molecule & Catalog \\
\hline WX-Psc & 01:06:25.98 & $+12: 35: 53.0$ & $\mathrm{SiO}$ & APEX SHeFI \\
\hline $\mathrm{R}-\mathrm{Scl}$ & $01: 26: 58.079$ & $-32: 32: 35.76$ & $\mathrm{HCN}$ & APEX SHeFI \\
\hline o-Ceti & 02:19:20.790 & $-02: 58: 41.91$ & $\mathrm{SiO}$ & APEX SHeFI \\
\hline R-For & $02: 29: 15.325$ & $-26: 05: 55.65$ & $\mathrm{HCN}$ & APEX SHeFI \\
\hline R-Hor & 02:53:52.899 & $-49: 53: 22.28$ & $\mathrm{SiO}$ & APEX SHeFI \\
\hline IK-Tau & $03: 53: 28.89$ & $+11: 24: 21.8$ & $\mathrm{SiO}$ & APEX SHeFI \\
\hline U-Men & 04:09:35.892 & $-81: 51: 17.57$ & $\mathrm{SiO}$ & SIMBAD, Optical \\
\hline R-Dor & $04: 36: 45.499$ & $-62: 04: 38.51$ & $\mathrm{SiO}$ & APEX SHeFI \\
\hline CRL618 & $04: 42: 53.64$ & $+36: 06: 53.4$ & $\mathrm{HCN}$ & APEX SHeFI \\
\hline R-Lep & $04: 59: 36.353$ & $-14: 48: 22.53$ & $\mathrm{HCN}$ & APEX SHeFI \\
\hline W-Ori & $05: 05: 23.723$ & $+01: 10: 39.46$ & $\mathrm{HCN}$ & APEX SHeFI \\
\hline U-Dor & 05:10:08.845 & $-64: 19: 04.27$ & $\mathrm{SiO}$ & SIMBAD, Optical \\
\hline $\mathrm{S}$-Pic & $05: 10: 57.248$ & $-48: 30: 25.45$ & $\mathrm{SiO}$ & SIMBAD, Optical \\
\hline R-Oct & 05:26:06.196 & $-86: 23: 17.77$ & $\mathrm{SiO}$ & SIMBAD, Optical \\
\hline V370-Aur & $05: 43: 49.68$ & $+32: 42: 06.2$ & $\mathrm{HCN}$ & APEX SHeFI \\
\hline N2071IR & $05: 47: 04.85$ & $+00: 21: 47.1$ & $\mathrm{HCN}$ & APEX LABOCA \\
\hline RAFGL865 & 06:04:00.0 & $+07: 25: 53.0$ & $\mathrm{HCN}$ & APEX SHeFI \\
\hline V636-Mon & $06: 25: 01.43$ & $-09: 07: 15.9$ & $\mathrm{HCN}$ & APEX SHeFI \\
\hline GX-Mon & $06: 52: 47.05$ & $+08: 25: 18.7$ & $\mathrm{SiO}$ & APEX SHeFI \\
\hline R-Vol & $07: 05: 36.20$ & $-73: 00: 52.0$ & $\mathrm{HCN}$ & APEX SHeFI \\
\hline L2-Pup & $07: 13: 32.42$ & $-44: 38: 19.8$ & $\mathrm{SiO}$ & APEX SHeFI \\
\hline HD56126 & $07: 16: 10.260$ & $+09: 59: 47.93$ & $\mathrm{HCN}$ & APEX SHeFI \\
\hline SVS-03513 & 07:17:05.68 & $-34: 49: 39.0$ & $\mathrm{SiO}$ & SEST \\
\hline VY-CMa & $07: 22: 58.321$ & $-25: 46: 03.49$ & $\mathrm{SiO}$ & APEX SHeFI \\
\hline $\mathrm{OH} 231.8$ & $07: 42: 16.92$ & $-14: 42: 50.0$ & $\mathrm{HCN}$ & APEX SHeFI \\
\hline 07454-7112 & $07: 45: 02.41$ & $-71: 19: 45.7$ & $\mathrm{HCN}$ & APEX SHeFI \\
\hline RAFGL1235 & $08: 10: 48.90$ & $-32: 52: 05.5$ & $\mathrm{HCN}$ & APEX SHeFI \\
\hline RAFGL5254 & $09: 13: 53.95$ & $-24: 51: 25.2$ & $\mathrm{HCN}$ & APEX SHeFI \\
\hline R-Car & $09: 32: 14.601$ & $-62: 47: 19.97$ & $\mathrm{SiO}$ & SIMBAD, Optical \\
\hline R-LMi & 09:45:34.293 & $+34: 30: 42.75$ & $\mathrm{SiO}$ & APEX SHeFI \\
\hline R-Leo & $09: 47: 33.494$ & $+11: 25: 43.21$ & $\mathrm{SiO}$ & APEX SHeFI \\
\hline IRC+10216 & 09:47:57.41 & $+13: 16: 43.6$ & $\mathrm{HCN}$ & APEX SHeFI \\
\hline RW-LMi & $10: 16: 02.29$ & $+30: 34: 19.1$ & $\mathrm{HCN}$ & APEX SHeFI \\
\hline U-Hya & $10: 37: 33.300$ & $-13: 23: 04.74$ & $\mathrm{HCN}$ & APEX SHeFI \\
\hline eta Carina & $10: 45: 03.53$ & $-59: 41: 03.75$ & Continuum & APEX LABOCA \\
\hline V-Hya & $10: 51: 37.248$ & $-21: 15: 00.38$ & $\mathrm{HCN}$ & APEX SHeFI \\
\hline R-Crt & $11: 00: 33.828$ & $-18: 19: 29.6$ & $\mathrm{SiO}$ & APEX SHeFI \\
\hline X-Cen & $11: 49: 11.788$ & $-41: 45: 27.27$ & $\mathrm{SiO}$ & SIMBAD, Optical \\
\hline Y-Cvn & $12: 45: 07.828$ & $+45: 26: 24.92$ & $\mathrm{HCN}$ & SIMBAD, Optical \\
\hline RT-Vir & $13: 02: 38.007$ & $+05: 11: 08.24$ & $\mathrm{SiO}$ & APEX SHeFI \\
\hline W-Hya & 13:49:01.961 & $-28: 22: 04.13$ & $\mathrm{SiO}$ & APEX SHeFI \\
\hline RX-Boo & $14: 24: 11.644$ & $+25: 42: 12.93$ & $\mathrm{SiO}$ & APEX SHeFI \\
\hline RAFGL4211 & $15: 11: 41.45$ & $-48: 19: 59.0$ & $\mathrm{HCN}$ & APEX SHeFI \\
\hline $\mathrm{X}-\operatorname{Tr} \mathrm{A}$ & $15: 14: 19.180$ & $-70: 04: 46.17$ & $\mathrm{HCN}$ & APEX SHeFI \\
\hline $\mathrm{S}-\mathrm{CrB}$ & $15: 21: 23.947$ & $+31: 22: 02.39$ & $\mathrm{SiO}$ & APEX SHeFI \\
\hline IRAS15194-5115 & $15: 23: 05.07$ & $-51: 25: 58.7$ & $\mathrm{HCN}$ & APEX SHeFI \\
\hline IRSV1540 & $15: 44: 39.803$ & $-54: 23: 05.03$ & $\mathrm{SiO}$ & SIMBAD, Infrared \\
\hline NGC6072 & $16: 12: 58.079$ & $-36: 13: 46.06$ & $\mathrm{HCN}$ & APEX SHeFI \\
\hline U-Her & $16: 25: 47.472$ & $+18: 53: 32.86$ & $\mathrm{SiO}$ & SIMBAD, Optical \\
\hline IRAS16293 & $16: 32: 22.56$ & $-24: 28: 31.8$ & $\mathrm{HCN}$ & SIMBAD, Infrared \\
\hline RAFGL1922 & 17:07:58.11 & $-24: 44: 31.2$ & $\mathrm{HCN}$ & APEX SHeFI \\
\hline AH-Sco & $17: 11: 17.021$ & $-32: 19: 30.71$ & $\mathrm{SiO}$ & SIMBAD, Optical \\
\hline NGC6302 & $17: 13: 44.46$ & $-37: 06: 10.7$ & $\mathrm{HCN}$ & APEX SHeFI \\
\hline
\end{tabular}


Table B.1. continued.

\begin{tabular}{llllc}
\hline \hline Name & \multicolumn{1}{c}{ RA [hms] } & \multicolumn{1}{c}{ Dec $\left[^{\left.\circ{ }^{\prime \prime}\right]}\right.$} & Molecule & Catalog \\
\hline V2108-Oph & $17: 14: 19.393$ & $+08: 56: 02.60$ & $\mathrm{SiO}$ & SIMBAD, Infrared \\
IRC+20326 & $17: 31: 55.30$ & $+17: 45: 21.0$ & $\mathrm{HCN}$ & APEX SHeFI \\
OH2.6-0.4 & $17: 53: 18.92$ & $-26: 56: 37.1$ & $\mathrm{SiO}$ & SEST \\
VX-Sgr & $18: 08: 04.048$ & $-22: 13: 26.63$ & $\mathrm{SiO}$ & SIMBAD, Optical \\
RAFGL2135 & $18: 22: 34.68$ & $-27: 06: 29.4$ & $\mathrm{HCN}$ & APEX SHeFI \\
RAFGL2155 & $18: 26: 05.84$ & $+23: 28: 46.7$ & $\mathrm{HCN}$ & APEX SHeFI \\
V1111-Oph & $18: 37: 19.26$ & $+10: 25: 42.2$ & $\mathrm{SiO}$ & APEX SHeFI \\
IRC+20370 & $18: 41: 54.55$ & $+17: 41: 08.6$ & $\mathrm{HCN}$ & APEX SHeFI \\
IRC+00365 & $18: 42: 24.87$ & $-02: 17: 27.2$ & $\mathrm{HCN}$ & APEX SHeFI \\
IRC-30398 & $18: 59: 13.851$ & $-29: 50: 20.44$ & $\mathrm{HCN}$ & SIMBAD, Optical \\
V-Aql & $19: 04: 24.162$ & $-05: 41: 05.43$ & $\mathrm{HCN}$ & APEX SHeFI \\
R-Aql & $19: 06: 22.254$ & $+08: 13: 47.25$ & $\mathrm{SiO}$ & APEX SHeFI \\
W-Aql & $19: 15: 23.365$ & $-07: 02: 50.36$ & $\mathrm{HCN}$ & APEX SHeFI \\
IRC-10502 & $19: 20: 18.12$ & $-08: 02: 12.0$ & $\mathrm{HCN}$ & APEX SHeFI \\
V1302-Aql & $19: 26: 48.03$ & $+11: 21: 16.7$ & $\mathrm{HCN}$ & APEX SHeFI \\
V1965-Cyg & $19: 34: 10.05$ & $+28: 04: 08.5$ & $\mathrm{HCN}$ & APEX SHeFI \\
GY-Aql & $19: 50: 06.329$ & $-07: 36: 52.52$ & $\mathrm{SiO}$ & APEX SHeFI \\
chi-Cyg & $19: 50: 33.905$ & $+32: 54: 50.19$ & $\mathrm{SiO}$ & APEX SHeFI \\
S-Pav & $19: 55: 13.967$ & $-59: 11: 44.34$ & $\mathrm{SiO}$ & SIMBAD, Optical \\
RAFGL2477 & $19: 56: 48.45$ & $+30: 44: 02.6$ & $\mathrm{HCN}$ & APEX SHeFI \\
RR-Aql & $19: 57: 36.044$ & $-01: 53: 11.80$ & $\mathrm{SiO}$ & APEX SHeFI \\
IRC-10529 & $20: 10: 27.87$ & $-06: 16: 13.6$ & $\mathrm{SiO}$ & APEX SHeFI \\
X-Pav & $20: 11: 45.896$ & $-59: 56: 12.95$ & $\mathrm{SiO}$ & APEX SHeFI \\
NML-Cyg & $20: 46: 25.54$ & $+40: 06: 59.4$ & $\mathrm{SiO}$ & SIMBAD, Infrared \\
CRL2688 & $21: 02: 18.78$ & $+36: 41: 37.75$ & $\mathrm{HCN}$ & APEX SHeFI \\
RV-Aqr & $21: 05: 51.74$ & $-00: 12: 42.0$ & $\mathrm{HCN}$ & SIMBAD, Infrared \\
EP-Aqr & $21: 46: 31.866$ & $-02: 12: 45.71$ & $\mathrm{SiO}$ & APEX SHeFI \\
pi1-Gru & $22: 22: 44.241$ & $-45: 56: 52.83$ & $\mathrm{SiO}$ & APEX SHeFI \\
S-Gru & $22: 26: 05.474$ & $-48: 26: 18.76$ & $\mathrm{SiO}$ & SIMBAD, Optical \\
RAFGL3068 & $23: 19: 12.61$ & $+17: 11: 33.1$ & $\mathrm{HCN}$ & APEX SHeFI \\
W-Peg & $23: 19: 50.501$ & $+26: 16: 43.66$ & $\mathrm{SiO}$ & SIMBAD, Optical \\
R-Aqr & $23: 43: 49.462$ & $-15: 17: 04.14$ & $\mathrm{SiO}$ & SIMBAD, Optical \\
\hline
\end{tabular}

